\title{
Expression and influence of pentraxin-3, HbAlc and ApoA1/ApoB in serum of patients with acute myocardial infarction combined with diabetes mellitus type 2
}

\author{
YANXIA ZHU ${ }^{1}$, XIAOFANG WANG $^{1}$, WEIHUA WANG ${ }^{2}$, HONGGUANG WANG $^{1}$ and FENGLEI ZHANG ${ }^{1}$ \\ Departments of ${ }^{1}$ Cardiology and ${ }^{2}$ Emergency, People's Hospital of Dongying, Dongying, Shandong 257091, P.R. China
}

Received December 19, 2017; Accepted February 15, 2018

DOI: $10.3892 /$ etm.2018.5930

\begin{abstract}
The present study investigated the clinical significance of changes in levels of hypersensitive plasma pentraxin-3 (PTX3), hemoglobin A1c (HbAlc) and apolipoprotein A-I (ApoA1)/apolipoprotein B (ApoB) on patients with acute myocardial infarction (AMI) and diabetes mellitus type 2 (T2DM). After admission, 100 patients diagnosed with AMI combined with T2DM were selected as Group A. According to the level of fasting blood glucose (FBG), they were then subdivided into Group A1 $(\mathrm{n}=44)$ with $\mathrm{FBG} \geq 13.0 \mathrm{mmol} / \mathrm{l}$ and Group A2 $(\mathrm{n}=56)$ with $\mathrm{FBG}<13.0 \mathrm{mmol} / \mathrm{l}$. A total of 100 hospitalized patients with AMI in People's Hospital of Dongying (Dongying, China) were collected as Group B, and 100 healthy people receiving physical examination in the Physical Examination Center of People's Hospital of Dongying were selected as Group C. Serum PTX3, HbAlc and ApoA1/ ApoB of all the study participants were tested, and diseased coronary artery vessels were divided into single-, double- and triple-vessel lesions according to their numbers. Logistic regression analysis was performed for the number of diseased coronary artery vessels and each index. The level of PTX3 in Group A1 was higher than that in Group A2; the level of ApoA1/ApoB in the former was lower than that in the latter $(\mathrm{P}<0.05)$; and the level of HbAlc in the former was significantly higher than that in the latter $(\mathrm{P}<0.01)$; the levels of PTX3 and HbA1c in Group A2 were higher than those in Group B, while the level of ApoA1/ApoB in the former was lower than that in the latter $(\mathrm{P}<0.05)$. Logistic regression analysis showed that the number of diseased coronary artery vessels was positively correlated with PTX3 and HbA1c, but negatively correlated with ApoA1/ApoB. PTX3, HbAlc and ApoA1/apoB have a certain clinical significance in assessing the severity of AMI combined with T2DM.
\end{abstract}

Correspondence to: Dr Yanxia Zhu, Department of Cardiology, People's Hospital of Dongying, 317 Dongchengnanyi Road, Dongying, Shandong 257091, P.R. China

E-mail: zp68et@163.com

Key words: pentraxin-3, HbAlc, ApoA1/ApoB, acute myocardial infarction, diabetes mellitus type 2

\section{Introduction}

Coronary heart disease is one of the most common cardiovascular diseases in China, and acute myocardial infarction (AMI) is the type with the highest lethality and the worst prognosis $(1,2)$. A study has shown that hyperglycemia plays an important role in the formation of atherosclerosis (AS), and investigations have shown a high prevalence of diabetes mellitus type 2 (T2DM) in patients with coronary heart disease (3). A series of chronic inflammatory reactions occur in the body of patients with AMI combined with T2DM. This process causes massive accumulation of macrophages, dendritic cells, mast cells and $\mathrm{T}$ cells, which promote arterial inflammation by releasing inflammatory cytokines, thus leading to the formation of coronary plaques $(4,5)$. Therefore, inflammatory cytokines in serum can be used to predict the formation of coronary plaques as well as their development and changes, and further used to monitor changes in coronary heart disease condition in patients and determine their prognoses (6-8). Hypersensitive plasma pentraxin-3 (PTX3) is a marker of inflammatory reaction and can be expressed in atherosclerotic plaques. PTX3 is closely related to endothelial cells, and the concentration of PTX3 in serum can reflect the inflammatory state of arteries in vivo (9). The abnormal lipid metabolism of T2DM is the most important cause of vascular complications in patients with DM. The change in the ratio of plasma apolipoprotein A1 (ApoA1) to apolipoprotein $\mathrm{B}$ (ApoB) can reflect the dynamic balance between anti-AS factors and pro-AS factors, and APoA1/ApoB can be regarded as a relatively good predictor for the onset risks of cardiovascular and cerebrovascular diseases (10). A study has shown that hemoglobin Alc (HbAlc) is a relatively good clinical indicator reflecting blood glucose control status, and it can also indirectly reflect the severity of DM in patients; elevated serum level of HbA1c will lead to increased risk of cardiovascular diseases such as MI (11).

In the present study, differences in serum levels of PTX3, HbAlc and ApoA1/ApoB in patients with MI combined with T2DM, patients only with MI and healthy people were detected, so as to compare the differences in the concentration of the three indexes in different numbers of diseased coronary artery vessels and different groups, analyzing the effects of PTX3, HbAlc and ApoA1/ApoB judging the severity of MI combined with T2DM in patients and evaluating the prognosis. 


\section{Materials and methods}

Study objects. A case-control study was conducted, in which 100 patients with AMI combined with T2DM hospitalized in the Department of Cardiology of People's Hospital of Dongying (Dongying, China) from January 2013 to August 2017 were selected as Group A. According to the level of fasting blood glucose (FBG), these patients were subdivided into Group A1 with FBG $\geq 13.0 \mathrm{mmol} / 1$ and Group A2 with FBG $<13.0 \mathrm{mmol} / \mathrm{l}$. A total of 100 hospitalized patients with AMI in People's Hospital of Dongying were collected as Group B, and 100 healthy people receiving physical examination in the Physical Examination Center of People's Hospital of Dongying were selected as Group C. The study was approved by the Ethics Committee of People's Hospital of Dongying (Dongying, China) and written informed consents were signed by the patients and/or guardians.

Diagnostic methods.Diagnostic criteria for AMI were according to the Diagnostic Criteria for Coronary Atherosclerotic Heart Disease in the 2010 Hygiene Standards of the People's Republic of China (12): i) patients with a clinical history of ischemic chest pain; ii) patients whose electrocardiogram showed dynamic evolution; and iii) patients with dynamic changes in the concentration of serum myocardial markers of myocardial necrosis. Patients meeting 2 or 3 criteria above can be diagnosed as AMI.

Diagnostic criteria for patients with DM were based on the criteria formulated by the World Health Organization in 1999 (13): patients showing DM symptoms with intravenous plasma $\mathrm{FBG} \geq 7.0 \mathrm{mmol} / 1$ at any time, random blood glucose $\geq 11.1 \mathrm{mmol} / 1$, or $2 \mathrm{~h}$ blood glucose $\geq 11.1 \mathrm{mmol} / 1$ shown in an oral glucose tolerance test (OGTT).

Patients with malignant tumors, acute and chronic infectious diseases, severe liver and kidney dysfunction, immune system diseases, DM type I or ketoacidosis, pregnant women and minors under 18 years old were excluded.

Data collection methods. Basic data of the participants were collected, including age, weight, height, sex, smoking history and blood pressure value. Fasting venous blood was collected after 12-h fasting, and the levels of PTX3, HbAlc and ApoA1/ApoB were measured.

The results of coronary angiography were collected. Divisions of the number of vascular lesions in patients in the AMI combined with T2DM group and the AMI group: A lesion involving any one of the anterior descending branch, circumflex branch, or right coronary artery was identified as a single-vessel lesion; a lesion involving any two of them was determined as a double-vessel lesion; a lesion involving all the three branches was judged as a triple-vessel lesion; the left main coronary artery lesion was identified as a triple-vessel lesion.

Detection methods. The concentration of PTX3 was determined by enzyme-linked immunosorbent assay (Gbd Group, Inc., Woodbridge, VA, USA), and those of ApoA1 and ApoB were determined by an automatic biochemical analyzer. The percentage of HbAlc was determined by ion exchange high-performance liquid chromatography (HPLC) automatic analyzer.
Statistical analysis. SPSS 13.0 software (SPSS, Inc., Chicago, IL, USA) was used for statistical analysis. Chi-square test was used for qualitative data, and partitioning of Chi-square was used for pairwise comparisons. The normally distributed quantitative data were analyzed by analysis of variance or t-test; pairwise comparisons were detected by StudentNewman-Keuls-Q (SNK-Q) test; the non-normally distributed quantitative data were detected by rank-sum test. The relationship of the number of diseased coronary artery vessels with each index was analyzed by Spearman's correlation analysis and logistic regression analysis. All P-values represented bilateral probability and significance level was set as 0.05 .

\section{Results}

In this study, a total of 100 patients with AMI combined with T2DM were enrolled. Among them, there were 44 patients with $\mathrm{FBG} \geq 13.0 \mathrm{mmol} / 1$ (Group A1) and 56 patients with FBG $<13.0 \mathrm{mmol} / 1$ (Group A2); there were 100 patients with AMI (Group B), and 100 healthy people in the Physical Examination Center (Group C).

Comparisons of basic data among each group. There were no statistical significant differences in sex, age and body mass index among Group A1, A2, B and C ( $>>0.05)$. The proportions of patients who smoked in Group A1, A2 and B were higher than that in Group $\mathrm{C}(\mathrm{P}<0.05)$; the systolic and diastolic blood pressure in Group A1 and A2 were significantly higher than those in Group B and $\mathrm{C}(\mathrm{P}<0.05)$; the level of FBG in Group A1 was higher than that in Group A2 $(\mathrm{P}<0.05)$; the level of FBG in Group A2 was higher than those in Group B and C $(\mathrm{P}<0.05)$; there was no statistical significant difference in $\mathrm{FBG}$ between Group B and C $(\mathrm{P}<0.05)$ (Table I).

Comparisons of PTX3, HbAlc and ApoAl/ApoB among the four groups of patients. PTX3 level was the highest in Group A1, followed by Group A2, Group B and Group C $(\mathrm{P}<0.05)$. HbAlc and ApoA1/ApoB were the lowest in Group A1, followed by Group A2 $(\mathrm{P}<0.05)$, but there was no significant difference between Group B and C ( $>0.05)$; HbAlc in Group A1 was significantly higher than that in Group A2 $(\mathrm{P}<0.01)$ (Table II).

Comparison of the numbers of diseased coronary artery vessels between the AMI combined with T2DM group and the AMI group. Comparisons of the numbers of diseased coronary artery vessels among Group A1, A2 and B showed that the number of diseased coronary artery vessels in Group A1 was significantly larger than those in Group $\mathrm{A} 2$ and $\mathrm{B}(\mathrm{P}<0.05)$; the number of diseased coronary artery vessels in Group A2 was the largest $(\mathrm{P}<0.05)$, followed by Group $\mathrm{A} 1$, and finally Group B; the numbers of single-vessel coronary artery lesions in Group A1 and A2 were smaller than that in Group B $(\mathrm{P}<0.05)$ (Table III).

Correlation of the number of diseased coronary artery vessels with PTX, HbAlc and ApoAl/ApoB. Spearman's rank correlation analysis showed that the number of diseased coronary artery vessels was positively correlated with PTX3 and HbAlc ( $\mathrm{r}=0.667, \mathrm{P}=0.001 ; \mathrm{r}=0.548, \mathrm{P}<0.008)$, but negatively correlated with ApoA1/ApoB ( $\mathrm{r}=-0.710, \mathrm{P}<0.001)$. Logistic 
Table I. Comparisons of basic data among each group (mean \pm SD).

\begin{tabular}{lcccc}
\hline Factors & A1 group $(\mathrm{n}=44)$ & A2 group $(\mathrm{n}=56)$ & B group $(\mathrm{n}=100)$ & C group $(\mathrm{n}=100)$ \\
\hline Sex (male/female) & $22 / 20$ & $29 / 26$ & $56 / 44$ & $51 / 49$ \\
Age (years) & $61.88 \pm 9.18$ & $62.09 \pm 10.25$ & $60.30 \pm 9.33$ & $59.89 \pm 11.50$ \\
Body mass index $\left(\mathrm{kg} / \mathrm{m}^{2}\right)$ & $24.91 \pm 3.21$ & $23.32 \pm 4.58$ & $23.97 \pm 3.87$ & $23.10 \pm 2.12$ \\
Smoking history $(\%)$ & $50.00^{\mathrm{c}}$ & $46.43^{\mathrm{c}}$ & $52.00^{\mathrm{c}}$ & 18.00 \\
SBP $(\mathrm{mmHg})$ & $148.08 \pm 15.28^{\mathrm{b}, \mathrm{c}}$ & $147.70 \pm 14.27^{\mathrm{b}, \mathrm{c}}$ & $126.50 \pm 13.68$ & $123.35 \pm 12.80$ \\
DBP $(\mathrm{mmHg})$ & $88.84 \pm 3.51^{\mathrm{b}, \mathrm{c}}$ & $86.04 \pm 4.25^{\mathrm{b}, \mathrm{c}}$ & $77.56 \pm 2.60$ & $76.69 \pm 1.46$ \\
FBG $(\mathrm{mmmol} / \mathrm{l})$ & $16.32 \pm 2.26^{\mathrm{a}-\mathrm{c}}$ & $8.66 \pm 3.57^{\mathrm{b}, \mathrm{c}}$ & $5.50 \pm 0.98$ & $5.28 \pm 0.81$
\end{tabular}

${ }^{\mathrm{a} C}$ Compared with Group A2, $\mathrm{P}<0.05$; ${ }^{\mathrm{b}}$ compared with Group B, $\mathrm{P}<0.05$; ${ }^{\mathrm{c}}$ compared with Group C, $\mathrm{P}<0.05$.

Table II. Comparisons of PTX3, HbAlc and ApoA1/ApoB among the four groups of patients (mean \pm SD).

\begin{tabular}{lcccc}
\hline Testing indexes & A1 group $(\mathrm{n}=44)$ & A2 group $(\mathrm{n}=56)$ & B group $(\mathrm{n}=100)$ & C group $(\mathrm{n}=100)$ \\
\hline PTX3 $(\mu \mathrm{g} / \mathrm{l})$ & $7.54 \pm 0.43^{\mathrm{a}-\mathrm{c}}$ & $6.68 \pm 0.57^{\mathrm{b}, \mathrm{c}}$ & $5.62 \pm 0.53^{\mathrm{c}}$ & $1.67 \pm 0.81$ \\
HbA1c $(\%)$ & $10.82 \pm 0.61^{\mathrm{d}-\mathrm{f}}$ & $8.50 \pm 0.57^{\mathrm{d}, \mathrm{e}}$ & $7.26 \pm 0.68$ & $6.59 \pm 0.62$ \\
ApoA1/ApoB & $1.12 \pm 0.23^{\mathrm{g}-\mathrm{i}}$ & $1.28 \pm 0.19^{\mathrm{g}, \mathrm{h}}$ & $1.49 \pm 0.30$ & $1.76 \pm 0.43$ \\
\hline
\end{tabular}

${ }^{\mathrm{a} C}$ Compared with Group A2, P<0.05; ${ }^{\mathrm{b}}$ compared with Group B, P<0.05; ${ }^{\mathrm{c}}$ compared with Group C, P<0.05; ${ }^{\mathrm{d}}$ compared with Group A2, P $<0.01$; ${ }^{\mathrm{e}}$ compared with Group B, P $<0.05$; ${ }^{\mathrm{f}}$ compared with Group C, P $<0.05$; ${ }^{\circ}$ compared with Group A2, P $<0.01$; ${ }^{\text {h}}$ compared with Group B, P $<0.05$; ${ }^{\mathrm{i}}$ compared with Group C, $\mathrm{P}<0.05$.

Table III. Comparison of the number of diseased coronary artery vessels between the AMI combined with T2DM group and the AMI group [n (\%)].

\begin{tabular}{lrrrr}
\hline & & \multicolumn{2}{c}{ Nos. of diseased coronary artery vessels } \\
\cline { 3 - 5 } Group & Cases & $1(\mathrm{n}=66)$ & $2(\mathrm{n}=76)$ & $3(\mathrm{n}=58)$ \\
\hline A1 group & 44 & $3(6.82)^{\mathrm{a}, \mathrm{b}}$ & $17(38.64)^{\mathrm{c}, \mathrm{d}}$ & $24(54.54)^{\mathrm{e}, \mathrm{f}}$ \\
A2 group & 56 & $8(14.29)^{\mathrm{b}}$ & $34(60.71)^{\mathrm{d}}$ & $14(25.00)$ \\
B group & 100 & $55(55.00)$ & $25(25.00)$ & $20(20.00)$ \\
\hline
\end{tabular}

${ }^{a}$ Compared with Group A2, $\mathrm{P}<0.05$; ${ }^{\mathrm{b}}$ compared with Group B, $\mathrm{P}<0.05$; ${ }^{c}$ compared with Group A2, $\mathrm{P}<0.05 ;{ }^{\mathrm{d}}$ compared with Group B, $\mathrm{P}<0.05$; ${ }^{\mathrm{e}}$ compared with Group A2, $\mathrm{P}<0.05$; ${ }^{\mathrm{f}}$ compared with Group B, $\mathrm{P}<0.05$.

regression analysis revealed that PTX3 and HbA1c were risk factors for the number of diseased coronary artery vessels [odds ratio $(\mathrm{OR})=2.575,95 \%$ confidence interval $(95 \% \mathrm{CI})$ : 1.717-3.433; OR=1.986, 95\% CI, 1.357-2.597], and ApoA1/ ApoB was a protective factor for the number of diseased coronary artery vessels $(\mathrm{OR}=0.186,95 \% \mathrm{CI}, 0.153-0.237)$ (Tables IV and V).

\section{Discussion}

The occurrence and development of coronary heart disease are closely related to atherosclerosis (AS), and AS is formed by a variety of inflammatory cytokines in the body involved in various factors. DM is an independent risk factor for patients
Table IV. Correlation analyses of the number of diseased coronary artery vessels with serum PTX3, HbA1c and ApoA1/ ApoB.

\begin{tabular}{lrr}
\hline & \multicolumn{2}{c}{$\begin{array}{c}\text { Nos. of diseased } \\
\text { coronary artery vessels }\end{array}$} \\
\cline { 2 - 3 } Factors & $\mathrm{r}$ & P-value \\
\hline PTX3 & 0.667 & 0.001 \\
HbA1c & 0.548 & 0.008 \\
ApoA1/ApoB & -0.710 & $<0.001$ \\
\hline
\end{tabular}

with coronary heart disease. In patients with coronary heart disease combined with T2DM, the persistent chronic hyperglycemia in the body damages the blood vessel wall and endothelial cells, and the action of a variety of inflammatory cytokines leads to the formation of atherosclerotic plaques, thus resulting in or accelerating the occurrence and development of coronary heart disease and other atherosclerotic lesions $(13,14)$. A number of studies in China and elsewhere indicated that there are correlations of serum PTX3, HbAlc and ApoA1/ApoB with the change in the condition of coronary heart disease or DM in patients and their prognoses (10-12). Therefore, in this study, serum levels of PTX3, HbAlc and ApoA1/ApoB in patients with AMI combined with T2DM were measured, and the effects of PTX3, HbAlc and ApoA1/ApoB in the diagnosis and prognosis evaluation of these patients were analyzed.

Pentraxin-3 (PTX3) is an inflammatory cytokine that is secreted by vascular endothelial cells and macrophages 
Table V. Logistic regression analyses of the number of diseased coronary artery vessels with serum PTX3, HbA1c and ApoA1/ ApoB.

\begin{tabular}{lcccrrr}
\hline Factors & B & SE & Wald & P-value & OR & $95 \%$ CI \\
\hline PTX3 & 0.946 & 0.438 & 5.893 & 0.012 & 2.575 & $1.717-3.433$ \\
HbA1c & 0.686 & 0.321 & 4.813 & 0.041 & 1.986 & $1.357-2.597$ \\
ApoA1/ApoB & -1.618 & 0.589 & 8.947 & $<0.001$ & 0.186 & $0.153-0.237$ \\
\hline
\end{tabular}

under the action of various inflammatory cytokines. PTX3 can be expressed in atherosclerotic plaques, and the increased concentration of it can reflect the activation and instability of atherosclerotic plaques; PTX-3 has been proved to be involved in the occurrence and development of many cardiovascular diseases $(10,15,16)$. The study revealed that the level of PTX3 in patients with AMI combined with T2DM in Group A1 was the highest, followed by patients with AMI combined with T2DM and AMI patients in Group A2, and the lowest level appeared in healthy people, indicating that patients with AMI combined with T2DM have the highest degree of inflammatory responses, severe vascular endothelial injuries and unstable atherosclerotic plaques.

HbAlc is a product of non-enzymatic glycation reaction of hemoglobin which occurs slowly and continuously under the action of hyperglycemia. The increase of HbAlc leads to the acceleration of vasoconstriction, which increases gene expression of chemokines and adhesion molecules, speeds up lymphocytes and monocytes transferring into the vessel wall, and promotes the formation of atherosclerotic plaques (17-19). HbAlc is a commonly used clinical indicator for glycemic control in DM, and it can indirectly reflect the severity of DM condition in patients. The increased HbAlc may increase the onset risk of cardiovascular disease $(20,21)$. It was found in this study that HbA1c level in Group A1 was significantly higher than that in Group A2, that in Group A2 was higher than that in Group B, and that in Group B had no difference with Group C, indicating that the higher the level of HbAlc in patients with coronary heart disease combined with MI, the more severe the DM condition will be.

ApoAl is the major apolipoprotein of high-density lipoprotein (HDL), which acts to transport cholesterol from the tissues of the body to the liver for catabolism, thereby preventing the deposition of cholesterol in the blood vessel wall. Therefore, ApoAl has the role of inhibiting the atherosclerotic plaque formation. ApoB is the major apolipoprotein of low-density lipoprotein cholesterol (LDL-C), and LDL-C, an integral part of atherosclerotic plaques, is a major risk factor for AS. Therefore, the increased $\mathrm{ApoB}$ is an indicator for predicting the high onset risk of coronary heart disease (22). The ratio of plasma ApoA1 to ApoB can indirectly reflect the balance between anti-AS factors and pro-AS factors in plasma (23-25). This study showed that ApoA1/ApoB in Group A1 was lower than that in Group A2, but there was no difference between Group B and C, indicating that the degree of AS in DM patients is relatively high, and the occurrence risk of cardiovascular events is also high.

In addition, Spearman's rank correlation analysis manifested that the number of diseased coronary artery vessels was positively associated with PTX3 and HbA1c, but negatively associated with ApoA1/ApoB. Multivariate linear regression analysis showed that PTX 3 and HbA1c were risk factors for the number of diseased coronary artery vessels, but ApoA1/ApoB was a protective factor for it, suggesting that with the increase in PTX3 and HbA1c and the decrease in ApoA1/ApoB in serum of AMI patients, the degree of coronary artery lesion will become more severe.

In summary, increased inflammatory responses (elevated PTX3), aggravated DM (elevated HbA1c) and lipid metabolism disorders (decreased ApoA1/ApoB) may occur in patients with AMI combined with T2DM. Therefore, PTX3, HbAlc and ApoA1/ApoB can be used to determine the severity of AMI combined with T2DM, assess the prognosis of patients and provide a basis for improving treatment programs.

\section{Acknowledgements}

Not applicable.

\section{Funding}

No funding was received.

\section{Availability of data and materials}

The datasets used and/or analyzed during the current study are available from the corresponding author on reasonable request.

\section{Authors' contributions}

YZ and XW conceived the study and drafted the manuscript. XW and WW collected the data from the patients. WW, HW and FZ analyzed and interpreted the data and revised the manuscript. All authors read and approved the final manuscript.

\section{Ethics approval and consent to participate}

This study was approved by the Ethics Committee of People's Hospital of Dongying (Dongying, China). Patients who participated in this research, signed the informed consent.

\section{Consent for publication}

Not applicable.

\section{Competing interests}

The authors declare that they have no competing interests. 


\section{References}

1. Tillmann T, Vaucher J, Okbay A, Pikhart H, Peasey A, Kubinova R, Pajak A, Tamosiunas A, Malyutina S, Hartwig FP, et al: Education and coronary heart disease: Mendelian randomisation study. BMJ 358: j3542, 2017.

2. Gilani N, Kazemnejad A, Zayeri F, Hadaegh F, Azizi F and Khalili D: Anthropometric indices as predictors of coronary heart disease risk: Joint modeling of longitudinal measurements and time to event. Iran J Public Health 46: 1546-1554, 2017.

3. Chen L, Wei B, Xu L and Wu Y: The association of inflammatory markers and periodontal indexes with the risk of coronary heart disease in Chinese patients with type 2 diabetes mellitus Diabetes Res Clin Pract 135: 37-44, 2017.

4. Kang HC and Hong JS: Association between costs and quality of acute myocardial infarction care hospitals under the Korea National Health Insurance program. Medicine (Baltimore) 96 : e7622, 2017.

5. Yavuz B, Kabakci G, Aksoy H, Tulumen E, Deveci OS, Aytemir K, Tokgozoglu L, Ozkutlu H, Nazli N and Oto A: Determining the relationship between metabolic syndrome score and angiographic severity of coronary artery disease. Int J Clin Pract 62: 717-722, 2008.

6. Wang J, Wu X, Li Y, Han X, Hu H, Wang F, Yu C, Li X, Yang K, Yuan J, et al: Serum bilirubin concentrations and incident coronary heart disease risk among patients with type 2 diabetes: The Dongfeng-Tongji cohort. Acta Diabetol 54: 257-264, 2017.

7. Farrokhian A, Bahmani F, Taghizadeh M, Mirhashemi SM, Aarabi MH, Raygan F, Aghadavod E and Asemi Z: Selenium supplementation affects insulin resistance and serum hs-CRP in patients with type 2 diabetes and coronary heart disease. Horm Metab Res 48: 263-268, 2016.

8. Ahmad S, Xue Z, Silverman A and Lindsay J: Complexity of the relation between hemoglobin $\mathrm{A} 1 \mathrm{C}$, diabetes mellitus, and progression of coronary narrowing in postmenopausal women. Am J Cardiol 111: 793-799, 2013.

9. Zhang J, Koussih L, Shan L, Halayko AJ, Chen BK and Gounni AS: TNF up-regulates Pentraxin3 expression in human airway smooth muscle cells via JNK and ERK1/2 MAPK pathways. Allergy Asthma Clin Immunol 11: 37, 2015.

10. Lu M, Lu Q, Zhang Y and Tian G: ApoB/apoA1 is an effective predictor of coronary heart disease risk in overweight and obesity. J Biomed Res 25: 266-273, 2011.

11. Claesson R, Ekelund M, Ignell C and Berntorp K: Role of HbA1c in post-partum screening of women with gestational diabetes mellitus. J Clin Transl Endocrinol 2: 21-25, 2014.

12. National standards of P.R.C. Diagnostic Criteria of Coronary Atherosclerotic Heart Disease. Beijing: Quality Inspection Press of China 1-4, 2010.

13. Deckers JG, Schellevis FG and Fleming DM: WHO diagnostic criteria as a validation tool for the diagnosis of diabetes mellitus: A study in five European countries. Eur J Gen Pract 12: 108-113, 2006.

14. Vigili de Kreutzenberg S, Solini A, Vitolo E, Boi A, Bacci S, Cocozza S, Nappo R, Rivellese A, Avogaro A and Baroni MG: Silent coronary heart disease in patients with type 2 diabetes: Application of a screening approach in a follow-up study. J Diabetes Complications 31: 952-957, 2017.
15. Emdin CA, Klarin D, Natarajan P, Florez JC, Kathiresan S, Khera AV and Khera AV; CARDIOGRAM Exome Consortium: Genetic variation at the sulfonylurea receptor, type 2 diabetes, and coronary heart disease. Diabetes 66: 2310-2315, 2017.

16. Naito A, Tanabe N, Jujo T, Shigeta A, Sugiura T, Sakao S, Ishida K and Tatsumi K: Pentraxin 3 in chronic thromboembolic pulmonary hypertension: A new biomarker for screening from remitted pulmonary thromboembolism. PLoS One 9: e113086, 2014.

17. Tarantino U, Feola M, Celi M and Scimeca M: PTX3: A new mediator of bone metabolism and osteoporosis. Muscles Ligaments Tendons J 7: 200-201, 2017.

18. Ohde S, Deshpande GA, Yokomichi H, Takahashi O, Fukui T and Yamagata Z: HbAlc monitoring interval in patients on treatment for stable type 2 diabetes. A ten-year retrospective, open cohort study. Diabetes Res Clin Pract 135: 166-171, 2018.

19. Hansel B, Giral P, Gambotti L, Lafourcade A, Peres G, Filipecki C, Kadouch D, Hartemann A, Oppert JM, Bruckert E, et al: A fully automated web-based program improves lifestyle habits and $\mathrm{HbAlc}$ in patients with type 2 diabetes and abdominal obesity: Randomized trial of patient E-coaching nutritional support (The ANODE Study). J Med Internet Res 19: e360, 2017.

20. Mousavi SN, Kamali K, Mirbazel M, Jameshorani M, Mousavi N, Kamali K, Mirbazel M and Jameshorani M: The best cut-off value for HbAlc as a screening tool in Iranian women with gestational diabetes mellitus. J Family Reprod Health 11: 37-42, 2017.

21. Jiang J, Zhao L, Lin L, Gui M, Aleteng Q, Wu B, Wang S, Pan B, Ling Y and Gao X: Postprandial blood glucose outweighs fasting blood glucose and HbA1c in screening coronary heart disease. Sci Rep 7: 14212, 2017.

22. Trivedi H, Gray LJ, Seidu S, Davies MJ, Charpentier G, Lindblad U, Kellner C, Nolan J, Pazderska A, Rutten G, et al: Selfknowledge of HbAlc in people with type 2 diabetes mellitus and its association with glycaemic control. Prim Care Diabetes 11: 414-420, 2017.

23. Bays HE, Shah A, Lin J, Sisk CM, Dong Q and Maccubbin D: Consistency of extended-release niacin/laropiprant effects on Lp(a), ApoB, non-HDL-C, Apo A1, and ApoB/ApoA1 ratio across patient subgroups. Am J Cardiovasc Drugs 12: 197-206, 2012.

24. Zheng J, Yin Q, Cao J and Zhang B: Obesity contributes more to increasing ApoB/ApoA1 ratio than hyperandrogenism in PCOS women aged 20-38 years in China. Exp Ther Med 13: 1337-1342, 2017.

25. Öhman M, Öhman ML and Wållberg-Jonsson S: The apoB/ apoA1 ratio predicts future cardiovascular events in patients with rheumatoid arthritis. Scand J Rheumatol 43: 259-264, 2014.

This work is licensed under a Creative Commons Attribution-NonCommercial-NoDerivatives 4.0 International (CC BY-NC-ND 4.0) License. 